\title{
Using Human-Centered Design Leadership to Improve Care of Older Adults With Disabilities
}

\author{
Daniel W. Eadens ${ }^{1} \&$ Danielle M. Eadens ${ }^{1}$ \\ ${ }^{1}$ Northern Arizona University, Flagstaff, USA \\ Correspondence: Daniel W. Eadens, EdD, Associate Professor, Educational Leadership Department, College of \\ Education's International Coordinator, Northern Arizona University, Flagstaff, Arizona, USA. Tel: 928-523-6242.
}

Received: August 26, 2018

Accepted: September 7, $2018 \quad$ Online Published: September 10, 2018

doi:10.5430/irhe.v3n3p65

URL: https://doi.org/10.5430/irhe.v3n3p65

\begin{abstract}
As the population of individuals with disabilities is rapidly aging, organizations face new challenges in person-centered care, especially in residential facilities where their caregivers are not family members but individuals with diverse cultural backgrounds and limited understanding of American culture. There are many organizations which offer a range of services to individuals with disabilities, including community-based residential settings where caregivers work with adults to increase independence in daily living skill development, behavior management, personal care, medication administration, and other services and activities. Complications arise when caregivers lack the knowledge, training, cultural understanding, and communication skills necessary to provide quality care. Much more skill acquisition is needed than the minimal State-required 40-hour orientation and training. This article outlines the leadership needs for specific studies that use human-centered designs to develop new trainings and work processes that are tailor-made to suit needs of caregivers and individuals for whom they care. Additionally, it discusses the need for studies that could lead to practice and policy changes needed based upon results of research to determine the levels of knowledge diverse caregivers, serving adults with disabilities, have regarding activities of daily living and the aging process.
\end{abstract}

Keywords: adult care, disabilities, aging, leadership, human-centered design

\section{Introduction}

\subsection{Problem}

Baby-boomers are aging and living much longer. It is estimated that $29 \%$ of all families living in the United States have a family member with a disability, which equates to about $19 \%$ of the population (Eidelman, 2012). In millions, it is about 3.3 with wheelchair needs, 10.2 in need of mobility aid(s), 1.8 with severe visual limitations, 1.0 with severe hearing impairments or identified as deaf, 2.5 with speech impairment, 16.1 with limited cognitive function, mental, emotional, developmental, or behavioral health disabilities and many also live in poverty (Eidelman, 2012). As can be imagined, it is normal that individuals with disabilities would want to strive to live as independently as possible and to make their own decisions and maximize self-autonomy and control. The government would prefer these individuals reside in typical housing with supports and progress toward supported employment (Eidelman, 2012). Although technology has increased, independent housing is often challenging, especially for the very elderly and those with disabilities. The main problem is that lack the of leadership initiating and conducting research studies in this field that could begin to better assess how institutions are using varied methods and human-centered designs to create and develop new trainings and work processes tailored to better enable caregivers. Clearly there is an urgent need for research to be conducted to determine the level of knowledge of diverse caregivers who serve adults with disabilities have regarding activities of daily living and the aging process.

\subsection{Legal}

There is a plethora of bills, acts, and federal legislation involved with disabilities and those numbers continue to grow. Of these, there are some landmark legislation decisions that establish the foundation in this area. This section attempts to outline a few of these to enable a better general understanding of how it affects facilities and institutions throughout the nation. For example, in 2000 the Developmental Disabilities Assistance and Bill of Rights Act was reauthorized and provides funding for each states' council on developmental disabilities, protection and advocacy 
systems, and a university center for excellence for individuals with developmental disabilities (Eidelman, 2012). This legislation was designed for individuals with lifetime disabilities that require assistance and supports to help with functional limitations. Independent living centers have an established funding formula because of the Amendments of the initial 1973 Rehabilitation Act and the reason for these centers are to "maximize the leadership, empowerment, independence, and productivity of individuals with disabilities and to integrate them into the mainstream of American society" (p. 4). The key in this legislation is inclusion and independent living training with advocacy and peer support. The 1990 Americans with Disabilities Act (ADA) is a civil rights law "prohibiting discrimination based on disability in employment, publicly funded services, and public accommodations" regardless of the severity of the individual's disability (p. 7).

\subsection{Improving Technology}

The Active Aging Research Center (AARC), housed in the University of Wisconsin-Madison's Center for Health Enhancement Systems Studies (CHESS), began its funding in 2010 from the federal agency for healthcare research (Gustafson, Maus, Judkins, Dinauer, Isham, Johnson, Landucci, \& Atwood, 2016). They developed technology specifically for older adults (aged 65 and older). CHESS worked with older adults, informal caregivers, health care professionals, and community members alike. Gustafson et al. (2016) claimed that "between 2008 and 2012, adults aged over 65 had an increase of 39\% in Internet use, the largest among all age groups" and " $50 \%$ of all older adults are online" now (p. 2). They believe older individuals might be even more willing to use more kinds of technologies if they felt that it "adds value and convenience to their lives and supports their activities" (p. 2). They also affirmed "designing a technology with and for older adults would help overcome barriers" (p. 2).

The Assistive Technology Act (ATA), first initiated in 1988, provides assistance "to persons with disabilities so they can more fully participate in education, employment, and daily activities on a level playing field with other members of their communities" (p. 6). It is designed to cover through the entire lifespan of the individual who has a disability within all aspects of those individuals' lives including, to mention only a few, assistance with wheelchairs, speech recognition devices, patient lifts, orthotics and prosthetics, hearing aids, smoke detectors, enlarging computer keyboards, amplified telephones, software, talking ATMS, voting machines for those who are blind, smart tablets and phones, etc. (Eidelman, 2012). Assistive Technology Act Projects (ATAP) are located in each state and is a location where individuals can find matching financial resources. "The statutory definition of Assistive Technology Device was amended into the following federal laws in order to assure the access to public funding when appropriate: IDEA, both Parts B and C; the Rehabilitation Act; and, the Developmental Disabilities Act. In most states, Medicaid can pay for a range of assistive technologies" (p. 6).

\subsection{Quality Supported Care}

Damen, Kef, Worm, Janssen, and Schuengel (2011, p. 581) claimed "the satisfaction that people with intellectual disabilities (IDs) have with their life is determined to a high degree by the quality and quantity of their interactions with other persons (e.g. Emerson \& Hatton 2008; Miller \& Chan 2008)". Continuing in that vein, Damen et al. (2011) said "Maes et al. suggest that improvements in the quality of interaction would be best made through a focus on both the clients, staff and the care organization" (p. 582). They conducted a study on "effective programme for supporting professional caregivers in improving the quality of interaction with clients with visual and intellectual disabilities" (p. 582). Damen et al. believed that caregiver's effective non-verbal communication skills and mutual affect, ideas, and activities are crucial to building strong quality client relationships. Interestingly, caregivers' work ethos, attitudes, and the "culture of their workplace" contribute to the "quality of interactions" (p. 582). Results from this study, despite limitations, indicated that "supporting professional care givers to increase their awareness of signals and skills for responding is followed by improved mutuality, affectivity and intimacy in interactions between caregivers and their clients" (p. 593). They recommended future research examine if more "explicit teaching of interactive skills leads to additional effects" (p. 593).

As life expectancy for those with severe disabilities increases, their needs for supports change. "Of the 75 percent of individuals with developmental disabilities who live at home with family, 25 percent live with caregivers who are over the age of sixty, and many are developing their own needs for support" (p. 35). When family caregivers become too elderly to care for family members with significant disabilities, they typically rely on professionals in family care facilities, and assume these facilities know and use best practice for their loved ones. These family planning conversations are often difficult ones for the families and facility managers. Still there remains much distrust for senior living facilities; despite the many outstanding facilities, it only takes a few bad reports to generalize and tarnish the whole. However, having a place and a plan is often comforting to families, especially during retirement 
and emergencies: knowing that resuscitation and end of life plans, legal, financial, and short and long-term care are settled can be very comforting for all parties (Eidelman, 2012).

Today, many senior living facilities and group care homes are understaffed, untrained, forced to respond to changing demographics, and lack cultural competence to deal with the aging constituents, possible addition of severe disabilities, and end of life issues. They need additional staff and professional development for their employees/caregivers. Often professional caregivers receive minimum wage and many of these employees only speak English as a second language. This can create countless communication and cultural barriers; much language support, professional development, and trainings are desperately needed.

\subsection{Communities}

The term normalizing is transitioning away from nursing homes, adult living facilities, and state-led institutions and into local communities, living in the presence of connections with family, with people with disabilities, and with supports. These is the goal for many. "Best practice in supporting people with disabilities in their communities is an evolving concept. It involves recognizing that support services, civil rights of people with disabilities, and opportunities for self-direction are integrally intertwined" to enjoy a higher quality of life (Eidelman, 2012, p. 26). However, this is not always possible or reasonable; there are many barriers. Today, nearly three-quarters of very senior citizens with disabilities live at home with their own families and adult children. This trend is continuing and is the only solution for many that cannot afford the high cost of safe senior living facilities. However, for those who are unable to live with their families, there are adult residential living facilities. Unfortunately, many of these facilities lack the necessary resources and training to maintain their programs and excellent care.

\section{Purpose of the Study}

There are segments in the general public, especially in public schools, that have shifted the paradigm to a foci on culturally responsive pedagogy (CRP). This is an approach that theoretically addresses mentor-subordinate relationships and connects content and methods to the individual interests and motivation (Gay, 2010). There is also a movement affront towards universal design for learning (UDL) which focuses on inclusive practices that spawn climates and instruction that allow students to engage with content through multiple learning styles and media and empowers them by making the curriculum accessible to all and holding each accountable through goal setting and choice (Meyer, Rose, \& Gordon, 2014). Regardless of specific paradigms, approaches or organizations, these types of methods are indicative of the myriad of real attempts of professional development movements that endeavor to close the gap between the needs of the constituents and the delivery of the professionals for the common good that realize today's demographic change and the serious changing needs of our citizens.

However, in dealing with aging adults with special needs in health care and adult living facilities across the nation, professional development and trainings for the caregivers themselves, for the most part, have not kept abreast to deal with the changing demographics and populations of the constituencies. There exists an ever-increasing gulf between them. When our population of individuals with disabilities age, organizations face new challenges in person-centered care. This is especially the case in residential facilities where caregivers are typically not family members, and sometimes are individuals with diverse cultural backgrounds and limited understanding of American culture. For example, a serious recent challenge is the development and provision of supports and services to adults in their population who are entering their senior years, many of them who are encountering the difficulties of various dementias as well as health-related issues affected by related genetic disabilities, e.g. Downs Syndrome, paired with the many others complications associated with aging.

Most communities have some sort of senior living facility of options for care these days. The facilities often circumspect around questions regarding whether they are indeed fully equipped and staffed with skilled caregivers. One such facility that is attempting to keep abreast is the Arizona Training and Evaluation Center (AZTEC). AZTEC is a facility that serves an area in the Southwest are of the US. This particular organization, like many, offers a wide range of services to individuals with disabilities, including community-based residential settings. More than 350 caregivers work with more than a hundred adult residents at AZTEC to increase their independence with daily living skill development, behavior management, personal care, medication administration, and other services and activities. Currently, AZTEC has about 130 residents who are served in a residential settings as well as various day programs. Approximately 79 of their residents are between 50 and 82 years of age. Four have Alzheimer's/Dementia and several others are identified as trending rapidly towards cognitive decline. Additionally, there are about 51 residents between the ages of 23 to 49 . Nevertheless, at this facility and many others, complications arise when the caregivers lack the knowledge, training, and communication skills necessary to provide quality care which requires more skill acquisition than the state-required 40-hour orientation training. 
The purpose of this review is to suggest more leadership in conducting studies that begin to assess how institutions are using human-centered designs and methods to develop new trainings and work processes that are tailored to fit the needs of caregivers and the individuals for whom they care. Specifically, this work discusses the urgent need for research to be conducted to determine the level of knowledge diverse caregivers serving adults with disabilities have regarding activities of daily living and the aging process. Results of said studies could generate new information, knowledge, methods, procedures, and rehabilitation techniques that leaders could use to facilitate maximizing the skills and potential of health care workers in this field. Designs with full inclusion and integration could be employed to facilitate independent living, family support, and economic and social self-sufficiency of adults and individuals with mild to severe disabilities. Ultimately, results could help improve the effectiveness of services authorized under the Rehabilitation Act of 1973, as amended.

A worldwide movement promoting universal design as a support for independence and participation for people with disabilities has evolved in response to an expanding demographic and social reality. There are simply more people living with a wider array of disabilities than ever before and with some of the longest lifespans in history. Today's multidisciplinary teams are beginning to recognize it and should address this impending complex healthcare challenge, i.e., to provide quality supports that help older adults with disabilities age well, research informed training is needed for a workforce of caregivers with limited knowledge and diverse cultural backgrounds. These types of in-depth empirical qualitative and quantitative studies and research could fill gaps in the literature and training related to improving outcomes in the care of older adults with disabilities.

\section{Methods}

A series of quantitative, qualitative, and/or mixed methods studies are urgently needed to solve these issues. These and other in-depth studies should be done throughout the nation.

Current studies need:

1) to identify gaps in caregiving knowledge among workers providing services to older adults with disabilities living in group residences;

2) to employ user-centered design (UCD) (i.e., a framework of processes in which caregivers' needs, wants, and limitations are given extensive attention at each stage of the program design process) to fill gaps in training for workers providing care to older adults with disabilities;

3) to pilot test and evaluate effectiveness of the training on caregivers (e.g., change in knowledge, work processes, experience of competence, caregiver satisfaction, and quality of care for older adults with disabilities);

4) to refine the enhanced training program based on evaluations; and

5) to disseminate findings, including promising practices, through professional presentations and publications.

Studies could have the overall aim of enhancing current caregiver training for workers with diverse backgrounds in discovering more effecting methods that could improve the care of older adults with disabilities, ultimately helping these adults age well. Research questions should strive towards identifying 1) caregivers' current level of knowledge: U.S. healthcare system, American wellness, nutrition, medication, the aging process, types of disabilities, and ADA practices; 2) caregivers' training needs and wants; and 3) human and technical barriers to effective training of caregivers with diverse backgrounds.

These kinds of research studies could take a multidisciplinary (human and organizational systems, medical anthropology, communications, curriculum and instructional design), multi-method (triangulation of data) approaches. It would be better if they occurred across the US and included urban and rural locations as well as be located in facilities not unlike AZTEC, in other nonprofit organization that provide residential care for adult individuals with disabilities.

\section{Sampling}

A convenience sampling at each site should be utilized for each study. The type of sampling and numbers needed must correspond to the type of study design. For quantitative analyses of each design, a priori sample size calculation should be used to determine the minimum $n$. For qualitative designs, small focus groups and individual interviews should be conducted to obtain real data the might enable the detection of themes of important patterns of helpful information. Pertinent rich information might be sorted for interpretations. 
In all cases, the actual employees who provide day-to-day care to older adults should be the target participants in the research. Well conducted in-depth studies similar to these described are not abundant, but are clearly needed to enhance policy and practice changes that are crucial as caregiver populations shift and senior adult populations with disabilities live longer and continue to increase exponentially.

\section{Resulting Significance and Impact}

With increases of populations of senior individuals with disabilities, organizations face new challenges in person-centered care, especially in residential facilities where their caregivers are not usually family members and are often have diverse cultural backgrounds with the possibility of limited understanding of English and American healthcare practices. Results of these kinds of studies could offer valuable information to leaders that employ caregivers who work with adults to increase their independence in daily living skills development, behavior management, personal care, medication administration, and other activities. Findings from these types of studies could enable more efficacious systems of professional development that could better equip caregivers, impart specific knowledge, training, and communication skills necessary to provide enhanced quality care, far beyond the marginal State-required orientations. Furthermore, conclusions from well-executed studies might augment care givers abilities and acumen with cultural understandings of the aging process. Findings and innovations from these varieties of research studies could change policies and help fill gaps in the literature and training methods related to improving outcomes in the care of adults with disabilities.

\section{References}

Alexander, R. (2011). Evidence, rhetoric and collateral damage: The problematic pursuit of 'world class' standards. Cambridge Journal of Education, 41(3), 265-286. https://doi.org/10.1080/0305764X.2011.607153

Bloomberg, K., West, D., \& Iacono, T. A. (2003). Picture it: An evaluation of a training program for careers of adults with severe and multiple disabilities. Journal of Intellectual and Developmental Disability, 28(3), 260-282.

Cobb, C. W., \& Douglas, P. H. (1928). A theory of production. American Economic Review, 18(1), 139-165. Retrieved from www.aeaweb.org/aer/top20/18.1.139-165.pdf

Coyle, C. E., Putman, M., Kramer, J., \& Mutchler, J. E. (2016). The role of aging and disability resource centers in serving adults aging with intellectual disabilities and their families: Findings from seven states. Journal of Aging \& Social Policy, 28(1), 1-14.

Damen, S., Kef, S., Worm, M., Janssen, M. J., \& Schuengel, C. (2011). Effects of video-feedback interaction training for professional caregivers of children and adults with visual and intellectual disabilities. Journal of Intellectual Disability Research, 55(6), 581-595.

Davies, S., Clarkson, P., Hughes, J., Stewart, K., Xie, C., Saunders, R., \& Challis, D. (2015). Resource allocation priorities in social care for adults with a learning disability: an analysis and comparison of different stakeholder perspectives. Tizard Learning Disability Review, 20(4), 199-206.

Duyar, I. (2006). Analyzing education productivity. Education Review, 9(4), 1-17. Retrieved from www.edrev.info/essays/v9n4index.html

Eidelman, S. M. (2012, November 1). A guide to funding disabilities and special needs. Jewish Funders Network. Retrieved https://www.jfunders.org/resources/philanthropic-resources/guide-funding-disabilities-and-special-needs

Emerson, E., \& Hatton, C. (2008). Self-reported well-being of women and men with intellectual disabilities in England. American Journal on Mental Retardation, 113, 143-55.

Field, A. (2009). Discovering statistics using SPSS. Thousand Oaks, CA: Sage.

Gay, G. (2010). Culturally responsive teaching. Teachers College Press: New York.

Gustafson, D.H. Jr., Maus, A., Judkins, J., Dinauer, S., Isham, A., Johnson, R., Landucci, G., \& Atwood, A.K. (2016). Using the NIATx model to implement user-centered design of technology for older adults. JMIR Human Factors, 3(1), e2. https://doi.org/10.2196/humanfactors.4853

Harris, D. N. (2007). Diminishing marginal returns and the production of education: An international analysis. Education Economics, 15(1), 31-53. https://doi.org/10.1080/09645290601133894 
Kelley, H., Siwatu, K.O., Tost, J. R., \& Martinez, J. (2015). Culturally familiar tasks on reading performance and self-efficacy of culturally and linguistically diverse students. Educational Psychology in Practice, 31, 293-313.

Ladson-Billings, G. (1995). But that's just good teaching! The case for culturally relevant pedagogy. Theory Into Practice, 34(3), 159-165.

Leake, D. W., Burgstahler, S., \& Izzo, M. V. (2011). Promoting transition success for culturally and linguistically diverse students with disabilities: The value of mentoring. Creative Education, 2(2), 121-129. https://doi.org/10.4236/ce.2011.22017

MacNeil, A. J., Prater, D. L., \& Busch, S. (2009). The effects of school culture and climate on student achievement. International Journal of Leadership in Education, 12(1), 73-84.

Maes B., Lambrechts G., Hostyn I., \& Petry K. (2007). Quality-enhancing interventions for people with profound intellectual and multiple disabilities: A review of the empirical research literature. Journal of Intellectual \& Developmental Disability, 32, 163-78.

Matson, J. L., \& Rivet, T. T. (2008). Characteristics of challenging behaviors in adults with autistic disorder, PDD-NOS, and intellectual disability. Journal of Intellectual and Developmental Disability, 33(4), 323-329.

McKernan, J., \& McKernan, J. (2013). Curriculum action research: A handbook of methods and resources for the reflective practitioner. London, UK: Routledge, Taylor \& Francis Group.

McNiff, J. (2013). Action research principles and practice (3rd ed.). London, UK: Routledge, Taylor \& Francis Group.

Meyer, A., Rose, D. H. R., \& Gordon, D. (2014). Universal design for learning: Theory and practice. CAST Professional Publishing.

Parsons, S., Daniels, H., Porter, J., \& Robertson, C. (2008). Resources, staff beliefs and organizational culture: Factors in the use of information and communication technology for adults with intellectual disabilities. Journal of Applied Research in Intellectual Disabilities, 21(1), 19-33.

Redfield, S. E., \& Kraft, T. (2012). What color is special education? Journal of Law \& Education, 41(1), 129-200. Retrieved from http://www.law.sc.edu/law/faculty_staff.shtml

Rose, D. H., \& Gravel, J. W. (2010). Universal design for learning. In E. Baker, P. Peterson, \& B. McGaw (Eds.). International Encyclopedia of Education (3rd ed.). Oxford: Elsevier.

Saats, C. (2014, May). Implicit racial bias and school discipline disparities. Exploring the Connection. Kirwan Institute, special report.

Savage, C., Hindlle, R. Meyer, L., Hynds, A., Penetito, W., \& Sleeter, C. (2011). Culturally responsive pedagogies in the classroom: indigenous student experiences across the curriculum. Asia-Pacific Journal of Teacher Education, 39, 183-198.

Seber, G., \& Lee, A. (2012). Linear regression analysis. Hoboken, NJ: John Wiley \& Sons. https://doi.org/10.1002/9780471722199.ch6

Snyder, T. D., \& Dillow, S. A. (2010). Digest of education statistics 2009 (NCES 2010-013). Washington, DC: National Center for Education Statistics, Institute of Education Sciences, US Department of Education. Retrieved from nces.ed.gov/pubs2011/2011015.pdf

Staats, C. (2014). Kieran institute report state of the science: Implicit bias review 2013. Retrieved from http://kirwaninstitute.osu.edu/docs/SOTS-Implicit_Bias.pdf_

Sullivan, A. L., \& Bal, A. (2013). Disproportionality in special education: Effects of individual and school variables on disability risk. Exceptional Children, 79(4), 475-494. Retrieved from http://www.cec.sped.org

Villegas, A., \& Lucas, T. (2002). Preparing culturally responsive teachers: Rethinking the curriculum. Journal of Teacher Education, 55, 20-32.

Willems, A., Embregts, P., Hendriks, L., \& Bosman, A. (2015, February). Towards a framework in interaction training for staff working with clients with intellectual disabilities and challenging behavior. Journal of Intellectual Disability Research, 60(2), 134-148.

Wright, P. W. D. (2004). The individuals with disabilities education improvement act of 2004: Overview, explanation and comparison idea 2004 v. IDEA 1997. Retrieved from www.wrightslaw.com 